\title{
Tratamento ECMO em pacientes com insuficiência respiratória por COVID-19 e melhora do quadro clínico
}

ECMO treatment on patients with respiratory failure because of COVID-19 and improvement of the clinical condition

Tratamiento con ECMO en pacientes con insuficiencia respiratoria por COVID-19 y mejoría del cuadro clínico

Virna Maria Lima Morais de Carvalho ORCID: https://orcid.org/0000-0002-1172-8238 Centro Universitário de Patos UNIFIP, Brasil E-mail: virnacarvalho@med.fiponline.edu.br Lana Beatriz de Oliveira Pinho Viana ORCID: https://orcid.org/0000-0001-8197-3223 Centro Universitário de Patos UNIFIP, Brasil E-mail: lanapinhov@gmail.com Andrea Vaz Diniz

ORCID: https://orcid.org/0000-0002-3865-9690 Centro Universitário de Patos UNIFIP, Brasil E-mail: andreadiniz15@gmail.com

Maria Eduarda Minervino Almeida ORCID: https://orcid.org/0000-0001-9105-9275 Centro Universitário de Patos UNIFIP, Brasil E-mail: dudaminervino2012@gmail.com

Divane Hannah Nóbrega de Melo ORCID: https://orcid.org/0000-0002-6986-7679 Centro Universitário de Patos UNIFIP, Brasil E-mail: divanehnm@gmail.com Dayana Macário Martins

ORCID: https://orcid.org/0000-0001-5959-9239 Centro Universitário de Patos UNIFIP, Brasil E-mail: dmacariomartins@gmail.com

Tiago Bruno Carneiro de Farias ORCID: https://orcid.org/0000-0002-0473-1244 Centro Universitário de Patos UNIFIP, Brasil E-mail: tiagofarias@ fiponline.edu.br

Milena Nunes Alves de Sousa

ORCID: https://orcid.org/0000-0001-8327-9147 Centro Universitário de Patos UNIFIP, Brasil E-mail: milenanunes@ fiponline.edu.br

\begin{abstract}
Resumo
A oxigenação por membrana extracorpórea (ECMO) tem sido amplamente utilizada em pacientes com insuficiência respiratória grave causada pelo novo coronavírus (COVID-19). Nessas pessoas, o interstício pulmonar é ocupado por células e substâncias inflamatórias, o que prejudica as trocas gasosas e causa hipoxemia. O tratamento com ECMO consiste em impulsionar o sangue do enfermo através de uma cânula para uma membrana artificial capaz de oxigenálo, que então está apto a retornar para o organismo. Objetivou-se identificar, em pacientes com insuficiência respiratória por COVID-19, se o tratamento ECMO comparado à terapêutica padrão proporciona melhoras no quadro clínico. Foi realizada, no mês de maio de 2021, uma revisão sistemática da literatura. A pesquisa foi realizada inicialmente em duas bases de dados: PubMed e BVS. Dos artigos resultantes, 7 foram selecionados por se encaixarem nos critérios de pesquisa. A partir da análise dos resultados, verificou-se que o tratamento com ECMO é capaz de reduzir a mortalidade dos pacientes que se encontram com insuficiência respiratória grave quando comparado aos outros tratamentos utilizados. Quando utilizado, esse recurso mostrou-se capaz de diminuir a quantidade de substâncias inflamatórias e a tempestade de citocinas, possibilitando, assim, a melhora no suprimento de oxigênio para os órgãos vitais e evitando lesões pulmonares decorrentes de danos mecânicos. Além disso, quando utilizada em conjunto com as medicações, mostrou redução da inflamação. Portanto, percebe-se que o ECMO é uma
\end{abstract}


boa alternativa aos pacientes, porém não quando usado isoladamente. É necessário complementar o tratamento com medicações que aliviem os sintomas.

Palavras-chave: Tratamento ECMO; Oxigenação por membrana extracorpórea; Insuficiência respiratória; COVID19; Eficácia clínica.

\begin{abstract}
Extracorporeal membrane oxygenation (ECMO) has been widely used in patients with severe respiratory failure caused by the new coronavirus (COVID-19). In those people, the pulmonary interstice is occupied by cells and inflammatory substances, which impairs gas exchange and causes hypoxemia. The ECMO treatment consists of boosting the blood of the person through a cannula to an artificial membrane capable of oxygenating it, which is then able to return to the body. The present study aimed to identify, on patients with respiratory failure due to COVID-19, whether the ECMO treatment compared to standard therapy provides improvements in the clinical condition. A systematic review of the literature was conducted in May 2021. The research was done in two databases: PubMed and BVS. From the resulting articles, 7 were selected because they fit the research criteria. From the analysis of the results, it was found that ECMO treatment is able to reduce the mortality of patients with severe respiratory failure when compared to other treatments. When used, this resource proved to be able to reduce the amount of inflammatory substances and the storm of cytokines, thus enabling improvement in the oxygen supply to vital organs and avoiding lung injuries resulting from mechanical damage. In addition, when used in conjunction with medications, it showed a reduction in inflammation. Therefore, it was perceived that ECMO is a good alternative to patients, but not when used alone. It is necessary to supplement treatment with medications that relieves the symptoms.
\end{abstract}

Keywords: ECMO treatment; Extracorporeal membrane oxygenation; Respiratory failure; COVID-19; Clinic efficacy.

\title{
Resumen
}

La oxigenación por membrana extracorpórea (ECMO) se ha utilizado ampliamente en pacientes con insuficiencia respiratoria grave causada por el nuevo coronavirus (COVID-19). En estas personas, el intersticio pulmonar está ocupado por células y sustancias inflamatorias, que dificultan el intercambio de gases y provocan hipoxemia. El tratamiento con ECMO consiste en empujar la sangre del paciente a través de una cánula hasta una membrana artificial capaz de oxigenarla, que luego es capaz de regresar al organismo. El objetivo fue identificar, en pacientes con insuficiencia respiratoria por COVID-19, si el tratamiento con ECMO en comparación con la terapia estándar proporciona mejoras en el cuadro clínico. En mayo de 2021 se realizó una revisión bibliográfica sistemática. La investigación se realizó inicialmente en dos bases de datos: PubMed y BVS. De los artículos resultantes, se seleccionaron 7 porque se ajustaban a los criterios de búsqueda. Del análisis de los resultados se encontró que el tratamiento con ECMO es capaz de reducir la mortalidad de los pacientes que se encuentran en insuficiencia respiratoria severa en comparación con otros tratamientos utilizados. Cuando se utilizó, este recurso demostró ser capaz de reducir la cantidad de sustancias inflamatorias y la tormenta de citocinas, permitiendo así una mejora en el suministro de oxígeno a los órganos vitales y previniendo lesiones pulmonares por daño mecánico. Además, cuando se usa junto con medicamentos, mostró una reducción de la inflamación. Por tanto, está claro que la ECMO es una buena alternativa para los pacientes, pero no cuando se usa sola. Es necesario complementar el tratamiento con medicamentos que alivien los síntomas.

Palabras clave: Tratamiento con ECMO; Oxigenación por membrana extracorpórea; Insuficiencia respiratória; COVID-19; Efectividad clínica.

\section{Introdução}

A doença do novo coronavírus (COVID-19) ou SARS-CoV-2 é um agravo que, na maioria das pessoas, manifesta sintomas leves e após alguns dias recuperam-se. O sistema imune, de modo geral, combate o vírus de forma efetiva. Contudo, em alguns casos, o vírus chega aos pulmões ocasionando sintomas graves, como falta de ar (Sousa, Estrela \& Bezerra, 2020).

Segundo Ribeiro (2021), circulação extracorpórea é um aparelho mecânico de uso temporário aplicado em casos de falência cardiorrespiratória. Tem como o objetivo ofertar suporte a órgãos vitais como o coração e o pulmão, permitindo a troca gasosa e a oxigenação sanguínea. Dessa forma, é utilizada em pacientes com insuficiência cardiopulmonar reversível desencadeada por doenças cardíacas, pulmonares ou outras causas infecciosas como, por exemplo, a pneumonia, tuberculose e influenza.

De acordo com Santos, Costa, Santos, Pereira e Santos (2016), a ECMO foi usada pela primeira vez em 1971 apresentando resultados variados, entretanto foi na epidemia de influenza A que se renovou o interesse por esse método de 
tratamento, que mostrou eficiência na melhoria da hipoxemia refratária em pacientes no mundo todo. Porém, apesar dos resultados extraordinários, essa terapia ainda é muito distante da realidade dos hospitais da rede pública brasileira, sobretudo pelo alto custo e pela necessitada de capacitação dos profissionais da área.

Romano, Mendes, Park e Costa (2017) comprovaram que muitos pacientes apresentaram insuficiência respiratória hipoxêmica grave e receberam suporte por meio de ECMO obtiveram taxas de sobrevida surpreendentemente altas (acima de $70 \%$ ) e não demonstraram sequelas graves relacionadas diretamente com o uso da ECMO. Contudo, esse tratamento não é indicado para todos os pacientes com SDRA, é contraindicada em pacientes com doenças crônicas disfuncionais, naqueles com disfunção aguda grave de múltiplos órgãos, pacientes com mais de 75 anos de idade (contraindicação relativa).

Kon et al. (2020) relatam que a utilização da terapia de ECMO em pacientes categorizados graves acometidos pela patologia de COVID-19 com importante comprometimento dos pulmões (acima de 75\%) e que tiveram indicação de terapia por ventilação mecânica obtiveram resultados importantes para sua recuperação, inclusive, conseguindo deambular pelo leitor e receberam alta hospitalar com funções neurológicas preservadas. Sendo assim, a ECMO é uma solução terapêutica importante para o tratamento de pacientes graves com COVID-19.

Este estudo tem como objetivo identificar, em pacientes com insuficiência respiratória por COVID-19, se o tratamento ECMO comparado à terapêutica padrão proporciona melhoras no quadro clínico.

\section{Metodologia}

Foi realizada uma revisão sistemática da literatura, a partir da execução de algumas etapas. Para a realização do presente estudo, adotou-se o método qualitativo, que consiste em uma pesquisa na qual a interpretação por parte dos autores sobre o fenômeno em questão é essencial para a construção da argumentação. O pesquisador é o principal instrumento de coleta de dados para a construção da pesquisa, sendo os dados coletados preferencialmente descritivos e a argumentação construída de forma narrativa no intuito de responder à questão de pesquisa (Parreira, Shitsuka, Parreira \& Shitsuka, 2018).

Inicialmente, foi elaborada a pergunta de pesquisa da revisão e nesta fase foi estabelecida a questão PICO, $\mathrm{P}=$ População (Pacientes com insuficiência respiratória por COVID-19), I = Intervenção (Tratamento ECMO), C = Controle ou comparação (Tratamento padrão para insuficiência respiratória), $\mathrm{O}=$ Desfecho (Melhora do quadro clínico). Então, o questionamento traçado foi "Em pacientes com insuficiência respiratória por COVID-19, o tratamento ECMO comparado à terapêutica padrão melhora o quadro clínico?".

Após a definição da questão PICO, foi feita a busca na literatura em Maio de 2021, em que se utilizaram as bases de dados da Biblioteca Virtual em Saúde (BVS) e Medical Publisher (PubMed) para pesquisar estudos que abordassem a questão da revisão. Tais plataformas foram escolhidas por serem de amplo acesso, de fácil uso, intuitivas e pelo número de publicações de acesso livre e gratuito. No processo de pesquisa das bases de dados, identificaram-se artigos que contemplassem a questão PICO em todas essas bases de dados.

A seleção dos artigos foi feita utilizando-se os Medical Subject Headings (MeSH) e a busca atendeu a seguinte combinação, a partir do operador booleano AND: "extracorporeal membrane oxygenation" AND "respiratory failure" AND COVID-19 AND effectiveness. Ressalta-se que esta associação restritiva se aplicou nos dois canais de busca. Além disso, incluíram-se ensaios clínicos randomizados, textos disponíveis completos e publicados em 2020 e 2021, pois o novo coronavírus surgiu no final de 2019. Foram excluídos os artigos que não se adequavam ao objetivo proposto e os repetidos em mais de uma base de dados, permanecendo pelo menos uma vez.

A extração dos dados, feita aos pares, buscou informações relacionadas com o tratamento ECMO em pacientes com insuficiência respiratória por COVID-19 e a melhora do quadro clínico desses pacientes e, a partir de então, efetivou-se a 
síntese dos dados em quadros. A síntese foi realizada por dois revisores e qualquer incongruência entre o material selecionado por ambos foi resolvida mediante discussões até chegar à decisão final.

O percurso de filtragem dos estudos que compõem esse artigo (Figura 1) adotou a Preferred Reporting Items for Systematic Reviews and Meta-Analyses ou a Recomendação PRISMA (McInnes et al., 2018).

Figura 1. Fluxograma de seleção do material com base na Recomendação PRISMA.

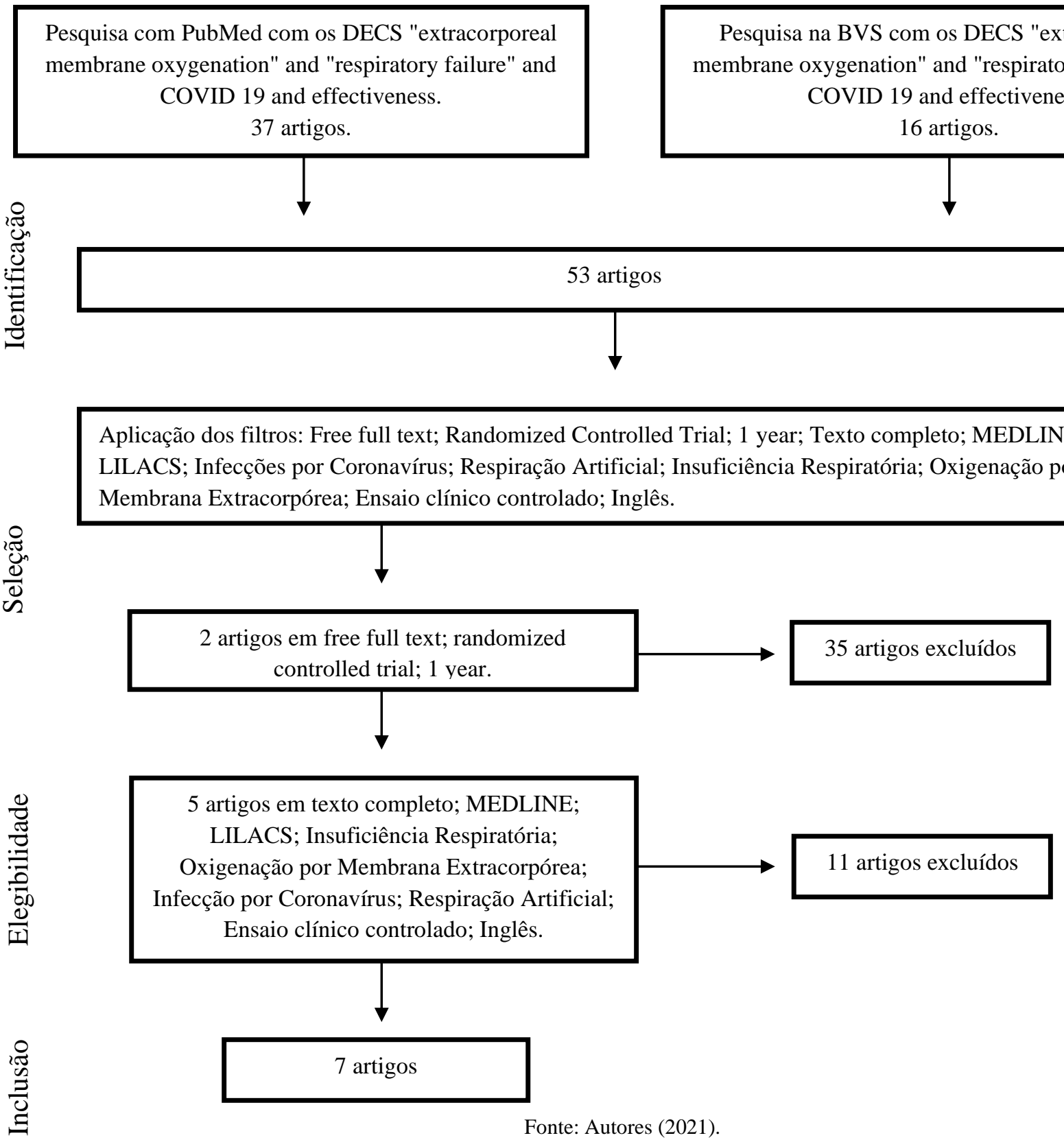

As informações selecionadas dos artigos foram: ano, país, tipo de estudo, nível de evidência, objetivo principal, grupo analisado, medicamentos, benefícios terapêuticos, tempo, conflito de interesse e efeitos adversos. A classificação da pirâmide de evidências utilizada foi a Oxford Centre for Evidence-Based Medicine (OCEBM) (Abrams, Khoury \& Grant, 2007). 


\section{Resultados e Discussão}

As publicações selecionadas foram realizadas em periódicos de excelência internacional 3A $(14,28 \%)$ e excelência nacional 1B (14,28\%) e 2B (57,14\%), conforme classificação Qualis Capes. Ademais, constata-se que a maioria dos estudos são oriundos da França, com o total de 2 artigos (28,57\% da amostra final).

Quadro 1: Caracterização geral dos artigos selecionados para compor a RIL. Patos, 2021.

\begin{tabular}{|c|c|c|c|c|}
\hline Autores & País & $\begin{array}{l}\text { Tipo de } \\
\text { estudo }\end{array}$ & $\begin{array}{l}\text { Nível de } \\
\text { evidência }\end{array}$ & Objetivo principal \\
\hline $\begin{array}{l}\text { Dequin et al. } \\
\text { (2020) }\end{array}$ & França & $\begin{array}{l}\text { Ensaio clínico } \\
\text { randomizado }\end{array}$ & $2 \mathrm{~B}$ & $\begin{array}{l}\text { Determinar o efeito da hidrocortisona na falha do tratamento no dia } \\
21 \text { em pacientes criticamente enfermos com infecção por síndrome } \\
\text { respiratória aguda grave por coronavírus } 2 \text { (SARS-CoV-2) e } \\
\text { insuficiência respiratória aguda. }\end{array}$ \\
\hline $\begin{array}{l}\text { Dioh et al. } \\
(2020)\end{array}$ & França & $\begin{array}{l}\text { Ensaio } \\
\text { controlado } \\
\text { randomizado }\end{array}$ & 1B & $\begin{array}{l}\text { Avaliar a segurança e eficácia do BIO101, cujo princípio ativo é a } \\
\text { 20-hidroxiecdysona, em pacientes COVID-19 com pneumonia grave. }\end{array}$ \\
\hline $\begin{array}{l}\text { Petersen et al. } \\
(2020)\end{array}$ & $\begin{array}{l}\text { Dinamarca, } \\
\text { Suécia, Suíça } \\
\text { e Índia }\end{array}$ & $\begin{array}{l}\text { Ensaio } \\
\text { controlado } \\
\text { randomizado }\end{array}$ & $2 \mathrm{~B}$ & $\begin{array}{l}\text { Avaliar os efeitos de baixas doses de Hidrocortisona versus Placebo } \\
\text { em pacientes adultos com COVID-19 e hipóxia grave. }\end{array}$ \\
\hline $\begin{array}{l}\text { Rilinger } \text { et al. } \\
\text { (2020) }\end{array}$ & Alemanha & $\begin{array}{l}\text { Ensaio } \\
\text { controlado } \\
\text { randomizado }\end{array}$ & $2 \mathrm{~B}$ & $\begin{array}{l}\text { Avaliar a eficácia e a segurança de um único tratamento de dose com } \\
\text { Tocilizumabe em pacientes com COVID-19 grave. }\end{array}$ \\
\hline $\begin{array}{l}\text { Roehrig et al. } \\
\text { (2020) }\end{array}$ & Qatar & $\begin{array}{l}\text { Ensaio clínico } \\
\text { randomizado }\end{array}$ & $2 \mathrm{~B}$ & $\begin{array}{l}\text { Demonstrar os efeitos positivos na oxigenação da ventilação } \\
\text { controlada por fluxo em comparação com pacientes ventilados } \\
\text { convencionalmente em pacientes que sofrem de síndrome do } \\
\text { desconforto respiratório agudo (SDRA) associado à COVID-19 }\end{array}$ \\
\hline $\begin{array}{l}\text { Shaefi et al. } \\
(2021)\end{array}$ & $\begin{array}{l}\text { Estados } \\
\text { Unidos }\end{array}$ & $\begin{array}{l}\text { Ensaio clínico } \\
\text { randomizado }\end{array}$ & $2 \mathrm{~B}$ & $\begin{array}{l}\text { Dados limitados estão disponíveis na oxigenação venosa da } \\
\text { membrana extracorpórea }(\mathrm{ECMO}) \text { em pacientes com insuficiência } \\
\text { respiratória hipoxêmica grave da doença coronavírus } 2019\end{array}$ \\
\hline $\begin{array}{l}\text { Verdejo et al. } \\
(2020)\end{array}$ & Colômbia & $\begin{array}{l}\text { Revisão } \\
\text { sistemática }\end{array}$ & $3^{a}$ & $\begin{array}{l}\text { Esta revisão viva e sistemática visa fornecer um resumo oportuno, } \\
\text { rigoroso e continuamente atualizado das evidências disponíveis sobre } \\
\text { o papel dos macrolídeos para o tratamento de pacientes com COVID- } \\
19 .\end{array}$ \\
\hline
\end{tabular}

Fonte: Autores (2021).

Os estudos selecionados foram analisados em grupos de pacientes contaminados com COVID-19, em sua maioria com sintomas graves e apresentando algum grau de desconforto respiratório. Foram utilizadas diferentes estratégias de tratamento, sendo: tratamento medicamentoso $(57,14 \%)$, ventilação mecânica + medicamento $(14,28 \%)$, ventilação controlada por fluxo $(14,28 \%)$ e oxigenação por membrana extracorpórea venovenosa - ECMO (14,28\%). Dentre os medicamentos utilizados, a hidrocortisona não mostrou eficácia significativa, porém quando associada à ventilação mecânica, conseguiu manter os pacientes por dias sem nenhum suporte de vida. O medicamento BIO101 apresentou propriedades que podem melhorar a função respiratória. Já o tocilizumabe foi utilizado esperando-se uma redução na dependência da ventilação 
mecânica e de internações em unidades de terapia intensiva. A combinação de macrolídeo + hidroxicloroquina não se mostrou eficaz. A ventilação controlada por fluxo mostrou efeitos positivos, como a melhora dos achados clínicos e radiológicos, assim como a ECMO, que mostrou mortalidade mais baixa do que os pacientes que não receberam.

Quadro2: Caracterização metodológica dos artigos selecionados para compor a RIL: grupo analisado, medicamentos e benefícios. Patos, 2021.

\begin{tabular}{|c|c|c|c|}
\hline $\begin{array}{l}\text { Autores/ } \\
\text { Ano }\end{array}$ & Grupo analisado & Medicamentos & Benefícios terapêuticos \\
\hline $\begin{array}{l}\text { Dequin et al. } \\
\text { (2020) }\end{array}$ & $\begin{array}{l}\text { Pacientes criticamente enfermos } \\
\text { com infecção por síndrome } \\
\text { respiratória aguda grave por } \\
\text { coronavírus } 2 \text { (SARS-CoV-2) e } \\
\text { insuficiência respiratória aguda. }\end{array}$ & $\begin{array}{l}\text { Uso de hidrocortisona em baixa } \\
\text { dose ou placebo. }\end{array}$ & $\begin{array}{l}\text { A hidrocortisona em baixa dose, em comparação } \\
\text { com o placebo, não reduziu significativamente a } \\
\text { falha do tratamento (definida como morte ou } \\
\text { suporte respiratório persistente) no dia } 21 \text {. }\end{array}$ \\
\hline $\begin{array}{l}\text { Dioh et al. } \\
(2020)\end{array}$ & $\begin{array}{l}\text { Pacientes acima de } 45 \text { anos } \\
\text { diagnosticados com COVID-19 } \\
\text { na forma mais grave pneumonia } \\
\text { grave. }\end{array}$ & $\begin{array}{l}\text { Uma linha intervencionista com } \\
\text { administração de } 350 \mathrm{mg} \mathrm{de} \\
\text { BIO101 (AP 20-hidroxiecdysona,) } \\
\text { e uma linha comparativa com uso } \\
\text { de } 350 \mathrm{mg} \text { de placebo. }\end{array}$ & $\begin{array}{l}\text { O BIO101(AP 20-hidroxiecdysona) apresenta } \\
\text { propriedades anti-inflamatórias, antifibróticas, } \\
\text { então pode oferecer uma melhora na função } \\
\text { respiratória, promovendo a sobrevivência dos } \\
\text { pacientes com COVID-19. }\end{array}$ \\
\hline $\begin{array}{l}\text { Petersen } \text { et al. } \\
(2020)\end{array}$ & $\begin{array}{l}1.000 \text { pacientes adultos com } \\
\text { COVID-19. }\end{array}$ & $\begin{array}{l}\text { Uma parte dos participantes desse } \\
\text { estudo receberam } \geq 10 \mathrm{~L} / \text { min de } \\
\text { oxigênio em ventilação mecânica } \\
\text { para hidrocortisona intravenosa } \\
200 \mathrm{mg} \text { por dia e a outra parte } \\
\text { receberam um placebo contendo } \\
\text { solução salina a } 0,9 \% \text {. }\end{array}$ & $\begin{array}{l}\text { Os pacientes que fizeram o uso de hidrocortisona } \\
\text { intravenosa } 200 \mathrm{mg} \text { por dia ficaram dias sem } \\
\text { suporte de vida (ou seja, ventilação mecânica, } \\
\text { suporte circulatório e terapia de substituição } \\
\text { renal) e obtiveram qualidade de vida relacionada } \\
\text { à saúde em } 1 \text { ano. }\end{array}$ \\
\hline $\begin{array}{l}\text { Rilinger et al. } \\
\text { (2020) }\end{array}$ & Pacientes com COVID-19 grave. & $\begin{array}{l}\text { Aplicação de } 8 \mathrm{mg} / \mathrm{kg} \text { de peso } \\
\text { corporal (PC) de tocilizumabe IV } \\
\text { uma vez imediatamente após a } \\
\text { randomização }(12 \mathrm{mg} / \mathrm{kg} \text { para } \\
\text { pacientes com <30 kg de peso } \\
\text { corporal; a dose total não deve } \\
\text { exceder } 800 \mathrm{mg} \text { ) E tratamento } \\
\text { convencional. Braço de controle: } \\
\text { Placebo (NaCl) intravenosa uma } \\
\text { vez imediatamente após a } \\
\text { randomização E o tratamento } \\
\text { convencional. }\end{array}$ & $\begin{array}{l}\text { Os resultados desejáveis seriam que o } \\
\text { tocilizumabe reduzisse o número de dias que os } \\
\text { pacientes ficam dependentes de ventilação } \\
\text { mecânica e reduzisse a capacidade de invasão da } \\
\text { assistência respiratória. Além disso, esse } \\
\text { tratamento pode resultar em menos internações } \\
\text { em unidades de terapia intensiva. }\end{array}$ \\
\hline $\begin{array}{l}\text { Roehrig et al. } \\
\text { (2020) }\end{array}$ & $\begin{array}{l}\text { Pacientes maiores de } 18 \text { anos que } \\
\text { sofrem de síndrome do } \\
\text { desconforto respiratório agudo } \\
\text { (SDRA) associada a COVID-19. }\end{array}$ & $\begin{array}{l}\text { Uso de ventilação controlada por } \\
\text { fluxo. }\end{array}$ & $\begin{array}{l}\text { O estudo visa demonstrar os efeitos positivos da } \\
\text { ventilação controlada por fluxo, como a melhora } \\
\text { dos achados radiológicos e clínicos. }\end{array}$ \\
\hline
\end{tabular}




\begin{tabular}{|l|l|l|l|}
\hline $\begin{array}{l}\text { Shaefi et al. } \\
(2021)\end{array}$ & $\begin{array}{l}190 \text { pacientes tratados com } \\
\text { ECMO dentro de } 14 \text { dias de } \\
\text { admissão na UTI com idade } \\
\text { mediana de 49 anos. }\end{array}$ & $\begin{array}{l}\text { Uso de oxigenação por membrana } \\
\text { extracorpórea } \\
\text { (ECMO). }\end{array}$ & $\begin{array}{l}\text { Os pacientes com insuficiência respiratória grave } \\
\text { venosa }\end{array}$ \\
$\begin{array}{l}\text { Verdejo et al. } \\
(2020)\end{array}$ & Pacientes com COVID-19. & $\begin{array}{l}\text { Uso de macrolídeos e uma } \\
\text { combinação de azitromicina com } \\
\text { mortalidade mais baixa do que aqueles que não } \\
\text { receberam. }\end{array}$ & $\begin{array}{l}\text { O uso de macrolídeos no tratamento de pacientes } \\
\text { com COVID 19 não mostrou efeitos benéficos } \\
\text { em comparação com o tratamento padrão. }\end{array}$ \\
\hline
\end{tabular}

Fonte: Autores (2021).

Não foram observados efeitos adversos significativos com os tratamentos: hidrocortisona, tocilizumabe, ventilação controlada por fluxo e macrolídeos. Em pacientes acima de 45 anos diagnosticados com COVID-19 na forma pneumonia grave ocorreu a deterioração do quadro respiratório considerando a necessidade de ventilação mecânica, oxigenação por membrana extracorpórea (ECMO) e oxigênio de alto fluxo definido como fornecimento de oxigênio a um fluxo maior ou igual a $16 \mathrm{~L} / \mathrm{min}$. Os corticosteroides apresentaram efeitos indesejáveis, incluindo maior tempo para a depuração viral. A mortalidade dos pacientes com insuficiência respiratória grave de COVID-19 que receberam ECMO foi de 34,6\%.

Quadro3: Caracterização metodológica dos artigos selecionados para compor a RIL: tempo, conflito de interesse e efeitos adversos. Patos, 2021

\begin{tabular}{|c|c|c|c|}
\hline $\begin{array}{l}\text { Autores/ } \\
\text { Ano }\end{array}$ & Tempo & Conflito de interesse & Efeitos adversos \\
\hline $\begin{array}{l}\text { Dequin et al. } \\
(2020)\end{array}$ & 21 dias. & $\begin{array}{l}\text { O Dr. Dequin relatou ter recebido bolsas da Abionic, Atox } \\
\text { Bio, Sphingotec GMBH, Adrenomed, Medspace, Aridis, } \\
\text { Merck, Combioxin, GlaxoSmithKline, MedImmune, } \\
\text { Genentech INH, RevImmune, Faron, Kenta e Tigenix. O } \\
\text { Dr. Voiriot relatou ter recebido honorários pessoais da } \\
\text { BioMérieux e receber bolsas da BioMérieux, SOS Oxygène } \\
\text { e Janssen. O Dr. Ricard relatou ter recebido doações da } \\
\text { Fisher \& Paykel. O Dr. Ehrmann relatou ter recebido } \\
\text { doações da Aerogen e Fisher \& Paykel; honorários pessoais } \\
\text { da Aerogen e La Diffusion Technique Française; e apoio } \\
\text { não financeiro da Aerogen, Fisher \& Paykel e La Diffusion } \\
\text { Technique Française. O Dr. Annane relatou ter recebido } \\
\text { uma bolsa do Ministério da Saúde da França, por meio do } \\
\text { Programa Nacional de Recherche Clinique, para } \\
\text { implementar a plataforma REMAPCAP na França, que tem } \\
\text { um domínio corticosteróide. }\end{array}$ & $\begin{array}{l}\text { Nenhum evento adverso sério foi } \\
\text { relacionado ao tratamento do } \\
\text { estudo. }\end{array}$ \\
\hline $\begin{array}{l}\text { Dioh et al. } \\
(2020)\end{array}$ & 28 dias & $\begin{array}{l}\text { Os autores declararam interesse não concorrente de que } \\
\text { todos os autores, exceto CMP, LAH, ML, TW e GBN, são } \\
\text { funcionários da BIOPHYTIS SA. Acreditam, no entanto, } \\
\text { que os potenciais interesses comerciais da empresa não } \\
\text { tiveram impacto na fundamentação científica ou na } \\
\text { condução do ensaio. }\end{array}$ & $\begin{array}{l}\text { Deterioração do quadro respiratório } \\
\text { considerando a necessidade de } \\
\text { ventilação mecânica, oxigenação } \\
\text { por membrana extracorpórea } \\
\text { (ECMO) e oxigênio de alto fluxo } \\
\text { definido como fornecimento de }\end{array}$ \\
\hline
\end{tabular}




\begin{tabular}{|c|c|c|c|}
\hline & & & $\begin{array}{l}\text { oxigênio a um fluxo maior ou igual } \\
\text { a } 16 \mathrm{~L} / \mathrm{min} \text {. }\end{array}$ \\
\hline $\begin{array}{l}\text { Petersen et } \\
\text { al. }(2020)\end{array}$ & 7 dias. & $\begin{array}{l}\text { O Dr. Friberg Hitz recebeu doações da OrklaCare, UCB, } \\
\text { Ellab Fond e Amgen, e recebeu pagamento pessoal em } \\
\text { relação a palestras e conselhos consultivos, nenhum dos } \\
\text { quais tem qualquer relação com o presente julgamento. Os } \\
\text { demais autores não têm conflito de interesses a declarar. }\end{array}$ & $\begin{array}{l}\text { Os corticosteroides têm efeitos } \\
\text { indesejáveis, incluindo maior tempo } \\
\text { para a depuração viral. }\end{array}$ \\
\hline $\begin{array}{l}\text { Rilinger et } \\
\text { al. (2020) }\end{array}$ & 8 meses & Os autores declaram não ter interesses conflitantes. & $\begin{array}{l}\text { Não foi mencionado no estudo } \\
\text { nenhum tipo de reação adversa ao } \\
\text { uso do tocilizumabe. }\end{array}$ \\
\hline $\begin{array}{l}\text { Roehrig et al. } \\
\text { (2020) }\end{array}$ & 4 meses. & Os autores declaram não ter interesses conflitantes. & $\begin{array}{l}\text { Não foi mencionado no estudo } \\
\text { nenhum tipo de reação adversa ao } \\
\text { uso da ventilação controlada por } \\
\text { fluxo. }\end{array}$ \\
\hline $\begin{array}{l}\text { Shaefi et al. } \\
(2021)\end{array}$ & $\begin{array}{l}\text { Os pacientes foram } \\
\text { acompanhados até a } \\
\text { alta hospitalar, óbito } \\
\text { ou um mínimo de } 60 \\
\text { dias. }\end{array}$ & $\begin{array}{l}\text { Os autores do comitê de redação são apoiados pelas } \\
\text { seguintes bolsas do National Institutes of Health. }\end{array}$ & $\begin{array}{l}34,6 \% \text { dos participantes que } \\
\text { receberam ECMO morreram. }\end{array}$ \\
\hline $\begin{array}{l}\text { Verdejo et al. } \\
\text { (2020) }\end{array}$ & $\begin{array}{l}\text { Não foi estabelecido } \\
\text { um tempo padrão para } \\
\text { os estudos analisados } \\
\text { nessa revisão. }\end{array}$ & Os autores não informação sobre os conflitos de interesse. & $\begin{array}{l}\text { Em relação aos efeitos adversos, } \\
\text { não houve diferença entre o } \\
\text { tratamento padrão e o uso de } \\
\text { macrolídeos. }\end{array}$ \\
\hline
\end{tabular}

Fonte: Autores (2021).

A partir da análise dos resultados dos artigos selecionados, foi verificado que a ECMO pode reduzir a mortalidade em pacientes com insuficiência respiratória grave devido ao COVID-19. Já no estudo do papel dos macrolídeos para o tratamento de pacientes com o vírus, houve resultados escassos de informações, sendo incapaz de obter conclusões precisas.

O grupo da hidrocortisona resultou em menos mortes do que o grupo do placebo. Já no tratamento da dose com tocilizumabe, o resultado esperado desse estudo é a redução do número de dias que o paciente depende de ventilação mecânica.

$\mathrm{O}$ artigo que estuda a eficácia do BIO101 resulta que a dificuldade respiratória requer ventilação mecânica, oxigenação por membrana extracorpórea (ECMO). A comparação com pacientes ventilados convencionalmente e pacientes com ventilação controlada por fluxo vão ser analisados a cada 6-8 horas, através de gravações. A análise do uso de corticoesteroides em COVID-19 e hipóxia grave obteve indicativos significativos na orientação do uso. 
Quadro4: Principais Resultados. Patos, 2021.

\begin{tabular}{|c|c|}
\hline Autores/ & Principais Resultados \\
\hline $\begin{array}{l}\text { Dequin et al. } \\
\text { (2020) }\end{array}$ & $\begin{array}{l}148 \text { pacientes }(99,3 \%) \text { completaram o estudo, houve } 69 \text { eventos de falha do tratamento, incluindo } 11 \\
\text { mortes no grupo da hidrocortisona e } 20 \text { mortes no grupo do placebo. }\end{array}$ \\
\hline $\begin{array}{l}\text { Dioh et al. } \\
\text { (2021) }\end{array}$ & $\begin{array}{l}\text { Dos resultados, requer ventilação mecânica, oxigenação por membrana extracorpórea (ECMO), } \\
\text { proporção de participantes com eventos "positivos" no Dia } 28 \text { e uma variedade de medidas funcionais e } \\
\text { biomarcadores, os quais deverão ser coletados. }\end{array}$ \\
\hline $\begin{array}{l}\text { Petersen } \text { et al. } \\
\text { (2020) }\end{array}$ & $\begin{array}{l}\text { O estudo COVID-STEROID fornece evidências importantes para orientar o uso de corticosteroides em } \\
\text { COVID-19 e hipóxia grave. }\end{array}$ \\
\hline $\begin{array}{l}\text { Rilinger et al. } \\
\text { (2020) }\end{array}$ & $\begin{array}{l}\text { Os resultados desejáveis são que o tocilizumabe reduza o número de dias que os pacientes ficam } \\
\text { dependentes de ventilação mecânica e a capacidade de invasão da assistência respiratória. }\end{array}$ \\
\hline $\begin{array}{l}\text { Roehrig et al. } \\
\text { (2020) }\end{array}$ & $\begin{array}{l}\text { O ponto final primário é } \mathrm{PaO} 2 \text {. Como este é um parâmetro dinâmico, iremos gravá-lo a cada 6-8 horas e } \\
\text { analisá-lo sequencialmente. }\end{array}$ \\
\hline $\begin{array}{l}\text { Shaefi et al. } \\
\text { (2021) }\end{array}$ & $\begin{array}{l}\text { Em pacientes selecionados com insuficiência respiratória grave de COVID-19, a ECMO pode reduzir a } \\
\text { mortalidade. }\end{array}$ \\
\hline $\begin{array}{l}\text { Verdejo et al. } \\
(2020)\end{array}$ & $\begin{array}{l}\text { Os resultados foram insuficientes para tirar conclusões válidas. A qualidade da evidência para os } \\
\text { resultados principais foi de baixa a muito baixa. }\end{array}$ \\
\hline
\end{tabular}

Fonte: Autores (2021).

\section{Discussão}

A partir dos estudos de Petersen et al. (2020), Dioh et al. (2020) e Shaefi et al. (2021) dessa revisão, foi possível constatar que a oxigenação por membrana extracorpórea (ECMO) possui pontos positivos e negativos, sendo importante avaliar os determinantes que predizem o sucesso ou a falha da terapia. Segundo Dequin et al. (2020), Rilinger et al. (2020) e Roehrig et al. (2020), outros tratamentos convencionais, como a hidrocortisona, o tocilizumab e a ventilação controlada por fluxo apresentaram resultados promissores. Já Verdejo et al. (2020) indicou que o uso de azitromicina e hidroxicloroquina possui baixas evidências para os principais resultados.

De acordo com Dequin et al. (2020), 148 pacientes (99,3\%) completaram o estudo, houve 69 eventos de falha do tratamento, incluindo 11 mortes no grupo da hidrocortisona e 20 mortes no grupo do placebo. Esse estudo foi fundamental para avaliar os prós e contras acerca do uso de corticoesteroides em adultos com COVID-19 e hipóxia grave. Em relação ao tratamento do estudo, não houve efeitos adversos que fossem considerados sérios. Ou seja, o grupo da hidrocortisona apresentou menos mortes do que o grupo do placebo, comprovando os resultados.

Segundo Petersen et al. (2020), em seu campo de estudo, fora constatado dois desfechos. No desfecho primário houve dias de vida sem a necessidade de suporte, ou seja, sem ventilação mecânica, suporte circulatório e terapia de substituição renal 
no $28^{\circ}$ dia de internação. Mas, no que diz respeito o desfecho secundário, indica que houve reações adversas graves no $14^{\circ}$ dia de internação. Sendo assim, o estudo torna-se contraditório tendo em visto que os resultados se contrapõem.

Acerca da oxigenação por membrana extracorpórea, Dioh et al. (2020) relatam que houve pontos positivos e negativos. Dentre os negativos tem-se deterioração respiratória e mortalidade por todas as causas no $28^{\circ}$, da mesma forma que também houve eventos positivos no mesmo dia, confirmando a tese de que os resultados dos estudos se contrapõem.

Shaefi et al. (2021) realizaram um estudo multicêntrico com 5.122 adultos em estado crítico com COVID-19. Destes, $190(3,7 \%)$ receberam o tratamento com ECMO e 127 (66,8\%) desses receberam alta hospitalar. Além disso, pacientes com hipoxemia grave que receberam ECMO nos primeiros 7 dias de internação apresentaram menor mortalidade hospitalar do que os que não fizeram. Com isso, foi constatado que o uso de ECMO, em pacientes com insuficiência respiratória grave, pode aumentar as chances de cura e reduzir a mortalidade. Dessa forma, apesar dos achados contraditórios encontrados em alguns estudos, de acordo com os resultados, foi verificado que a ECMO pode reduzir a mortalidade em pacientes com insuficiência respiratória grave devido ao COVID-19.

Haiduc, Alom, Melamed e Harky (2020) identificaram que muitos dos artigos com evidências contra ou inconclusivas em relação à ECMO para a COVID-19 têm uma pequena amostra e, por isso, não pode ser feita uma conclusão confiável. Além disso, muitos artigos não fornecem informações com relação à gravidade da doença do paciente no momento do início da ECMO, tornando inviável saber se a ECMO foi administrada em um momento tardio para ter um efeito significativo em um paciente grave com deterioração do quadro clínico. Também se pode destacar que muitos pacientes já tinham fatores de risco associados a uma alta taxa de mortalidade, como idade $\geq 60$ anos, comorbidades (doença cardiovascular e diabetes), baixa contagem de linfócitos $<0,8(\times 10 * 9 / \mathrm{L})$ e níveis de dímero $\mathrm{D}>1 \mu \mathrm{g} / \mathrm{L}$ em admissão, o que provavelmente foi determinante para o mau prognóstico desses pacientes.

Haiduc et al. (2020) também identificaram três fatores fundamentais para o sucesso da terapia ECMO para a COVID19: o momento precoce de suporte com ECMO, o reconhecimento e o tratamento da causa da deterioração da oxigenação e o papel da ECMO na redução de substâncias inflamatórias e aumento de sobrevida do paciente com associação entre COVID-19, tempestade de citocinas e mortalidade. Essas medidas possibilitaram a recuperação de pacientes, protegendo o suprimento de oxigênio de seus órgãos e evitando lesões pulmonares decorrentes de danos mecânicos (ventiladores), a estabilização da oxigenação e o descanso dos pulmões, além de mostrar resultados promissores na redução da inflamação quando utilizada em conjunto com o tratamento primário.

Em relação ao uso da droga tocilizumab, que é um inibidor da TNF-alfa e interleucina-6 que impede a tempestade de citocinas, um estudo realizado por Rilinger et al. (2020) afirmaram que o uso da mesma reduz o número de dias de internação de pacientes, com COVID-19 grave, fazendo uso de ventilação mecânica e a invasividade da assistência respiratória. Ainda, foi observado que essa medicação pode reduzir o número de internações em unidades de terapia intensiva. Concomitante a eficácia, constatou-se que a terapia com tocilizumab deve ser monitorada de perto, tendo em vista que a imunossupressão pode causar um aumento de casos de infecções bacterianas e influenciar de forma negativa a recuperação do paciente.

Além disso, outra classe de medicação também estudada no tratamento para COVID-19 foram os macrolídeos (Azitromicina). Verdejo et al. (2020) avaliaram os efeitos e desfechos desta como monoterapia ou de forma combinada com outras medicações em relação aos pacientes que não receberam esse tratamento, suspeitos ou confirmados por essa infecção viral. Atrelado a isso, estudou-se a necessidade de ventilação mecânica invasiva, oxigenação de membrana extracorpórea, tempo de internação hospitalar, insuficiência respiratória, eventos adversos graves e tempo de negatividade RT-PCR de SARSCoV-2. Neste mesmo experimento clínico randomizado, avaliou-se o uso associado de azitromicina com hidroxicloroquina, bem como o uso isolado de hidroxicloroquina e a partir disso, contatou-se que a qualidade das evidências para os principais 
resultados foi de baixa a muito baixa. Sendo assim, os desfechos foram insuficientes para se chegar a conclusões, o que foi indicado nos resultados.

Roehrig et al. (2020) em seu estudo sobre ventilação controlada por fluxo na Síndrome do Sofrimento Respiratório Agudo associada ao COVID-19, fizeram o monitoramento dos pacientes seguindo os critérios de inclusão que são: acima de 18 anos de idade, infecção comprovada por COVID-19, insuficiência respiratória que necessita de intubação e ventilação mecânica, ARDS com uma razão P/F de pelo menos 200mmHg ou menos e um PEEP mínimo 5cmH2O, IMC menos $30 \mathrm{~kg} /$ m2. Com base nisso, o cálculo do tamanho da amostra com base na suposição de um efeito (alteração na paO2) foi de 1,5 SDS no ponto final primário, um poder pretendido de $80 \%$, um erro alfa de 5\% e uma razão amostral resultando em n=7 pacientes necessários para tratar. De acordo com os resultados, foi observado que a ventilação controlada por fluxo mostrou efeitos positivos, como a melhora dos achados clínicos e radiológicos.

Por fim, é importante frisar as limitações desta revisão sistemática tendo em vista a busca de artigos em apenas duas bases de dados: BVS e PubMed. Além disso, são poucos os dados em relação à utilização de ECMO no COVID-19 devido aos estudos de pequeno porte, resultados limitados relatados e escassos estudos clínicos sobre o tema, o que impossibilita obter uma conclusão geral confiável sobre a utilização de ECMO para COVID-19. Também há uma deficiência de estudos no que se refere ao COVID-19, visto que a mesma é uma doença recente onde ainda não se sabe comprovadamente qual a terapia fixa eficaz para todos ou, pelo menos, para maioria dos casos. Juntamente a isso, é imprescindível que haja investimentos na saúde pública e no campo de pesquisa.

\section{Conclusão}

O uso de ECMO, em pacientes com insuficiência respiratória grave, pode aumentar as chances de cura e reduzir a mortalidade comparado à terapêutica padrão. O resultado dessa terapia depende de indicações precisas e análise detalhada da situação de cada paciente para garantir uma melhor sobrevida, como o momento precoce de suporte com ECMO, os fatores de risco dos pacientes, o reconhecimento e o tratamento da causa da deterioração da oxigenação e o papel da ECMO na redução de substâncias inflamatórias e aumento de sobrevida do paciente com associação entre COVID-19, tempestade de citocinas e mortalidade.

Outras terapias também indicaram resultados positivos, como a hidrocortisona que reduziu o número de mortes por COVID-19 grave; a droga tocilizumab que reduziu o número de dias de internação de pacientes com COVID-19 grave fazendo uso de ventilação mecânica e a invasividade da assistência respiratória ou em unidades de terapia intensiva. Já o uso associado de azitromicina com hidroxicloroquina, bem como o uso isolado de hidroxicloroquina tem evidências para os principais resultados de baixa a muito baixa. Por fim, foi observado que a ventilação controlada por fluxo mostrou efeitos positivos, como a melhora dos achados clínicos e radiológicos.

Portanto, observou-se a necessidade de estudos complementares futuros que permitam uma melhor avaliação do quadro clínico prévio do paciente de forma individualizada, visto que isso pode interferir na resposta à terapia ECMO. Além disso, faz-se necessária a compreensão do momento ideal para a intervenção da ECMO, já que essa terapia, se introduzida em um momento inadequado, pode causar danos e riscos à vida do paciente. Assim, é importante que sejam realizados mais estudos que esclareçam a funcionalidade da ECMO em aumentar a sobrevida das pessoas com COVID-19 em estado grave e se seus benefícios superam os riscos. 


\section{Referências}

Abrams, P., Khoury, S., \& Grant, A. (2007). Evidence-based medicine overview of the main steps for developing and grading guideline recommendations. Prog Urol, 17(3), 681-4. https://pubmed.ncbi.nlm.nih.gov/17622102/

Dequin, P. F., Heming, N., Meziani, F., Plantefève, G., Voiriot, G., Badié, J., François, B., et al. (2020). Effect of Hydrocortisone on 21-Day Mortality or Respiratory Support Among Critically Ill Patients With COVID-19: A Randomized Clinical Trial. JAMA, 324(13), 1-9. https://jamanetwork.com/journals/jama/fullarticle/2770276

Dioh, W., Chabane, M., Tourette, C., Azbekyan, A., Morelot-Panzini, C., Hajjar Lins, L. A. M., Nair, G. B., et al. (2020) Testing the efficacy and safety of BIO101, for the prevention of respiratory deterioration, in patients with COVID-19 pneumonia (COVA study): a structured summary of a study protocol for a randomised controlled trial. BMC Trials, 22(42). https://trialsjournal.biomedcentral.com/articles/10.1186/s13063-020-04998-5

Dos Santos, S. M., Costa, D. D. N., Santos, F. S., Pereira, R. S., \& Santos, E. S. (2016). Cuidado ao paciente em ECMO (Extracorporeal Membrane Oxygenation): um desafio para a enfermagem. Sempesq, 4(4), 64-68. https://eventos.set.edu.br/sempesq/article/view/4028

Grasselli, G., Zangrillo, A., Zanella, A., Antonelli, M., Cabrini, L., Castelli, A., Cereda D., et al. (2020). Baseline Characteristics and Outcomes of 1591 Patients Infected With SARS-CoV-2 Admitted to ICUs of the Lombardy Region, Italy. JAMA, 323(16), 1574-1581. https://jamanetwork.com/journals/jama/fullarticle/2764365

Haiduc, A. A., Alom, S., Melamed, N., \& Harky, A. (2020). Role of extracorporeal membrane oxygenation in COVID-19: a systematic review. Journal of Cardiac Surgery, 35(10), 2679-2687. https://pubmed.ncbi.nlm.nih.gov/32717771/

Huang, C., Wang, Y., Li, X., Ren, L., Zhao, J., Hu, Y., Zhang, L., et al. (2020). Clinical features of patients infected with 2019 novel coronavirus in Wuhan, China. Lancet, 395(10223), 497-506. https://www.thelancet.com/journals/lancet/article/PIIS0140-6736(20)30183-5/fulltext

Jacobs, J. P., Stammers, A. H., Louis, J. S., Hayanga, J. W. A., Firstenberg, M. S., Mongero, L. B., Tesdahl E. A., et al. (2020). Extracorporeal membrane oxygenation in the treatment of severe pulmonary and cardiac compromise in COVID-19: experience with 32 patients. ASAIO J, 66, $722-730$. https://pubmed.ncbi.nlm.nih.gov/32317557/

Kon, Z. N., Smith, D. E., Chang, S. H., Goldenberg, R. M., Angel, L. F., Carillo, J. A., Geraci, T. C., et al. (2020). Extracorporeal Membrane Oxygenation Support in Severe COVID-19. The Annals of Thoracic Surgery, 111(2), 537-543. https://pubmed.ncbi.nlm.nih.gov/32687823/

McInnes, M. D., Moher, D., Thombs, B. D., McGrath, T. A., Bossuyt, P. M., Clifford, T., \& Willis, B. H. (2018). Preferred reporting items for a systematic review and meta-analysis of diagnostic test accuracy studies: the PRISMA-DTA statement. JAMA, 319(4), 388-396. https://pubmed.ncbi.nlm.nih.gov/29362800/

Munshi, L., Walkey, A., Goligher, E., Pham, T., Uleryk, E. M., \& Fan E. (2019). Venovenous extracorporeal membrane oxygenation for acute respiratory distress syndrome: a systematic review and meta-analysis. Lancet Respir Med, 7, 163-172. https://pubmed.ncbi.nlm.nih.gov/30642776/

Pereira, A. S., Shitsuka, D. M., Parreira, F. J., \& Shitsuka, R. (2018). Metodologia da pesquisa científica. Santa Maria, RS: UFSM, NTE. https://repositorio.ufsm.br/bitstream/handle/1/15824/Lic_Computacao_Metodologia-Pesquisa-Cientifica.pdf?sequence=1

Petersen, M. W., Meyhoff, T. S., Helleberg, M., Kjær, M. B. N., Granholm, A., Hjortsø, C. J. S., Jensen, T. S., et al. (2020). Low-dose hydrocortisone in patients with COVID-19 and severe hypoxia (COVID STEROID) trial-Protocol and statistical analysis plan. Acta Anestesiológico Scand, 64(9), 1365-1375. https://pubmed.ncbi.nlm.nih.gov/32779728/

Ribeiro, A. P. R. (2020). ECMO A Terapia Que Salva Vidas: Revisão Sistemática. Revista Multidisciplinar e Psicologia, 54(14), 341-356. https://idonline.emnuvens.com.br/id/article/view/2974

Rilinger, J., Kern, W. V., Duerschmied, D., Supady, A., Bode, C., Staudacher, D. L., \& Wengenmayer, T. (2020). A prospective, randomised, double blind placebo-controlled trial to evaluate the efficacy and safety of tocilizumab in patients with severe COVID-19 pneumonia (TOC-COVID): A structured summary of a study protocol for a randomised controlled trial. Trials, 21, 470. https://trialsjournal.biomedcentral.com/articles/10.1186/s13063-020-04447-3

Roehrig, S., Hssain, A. A., Shallik, N. A. H., Elsaid, I. M. A., Mustafa, S. F., Smain, O. A. M., Molokhia, A. A., \& Lance, M. D. (2020). Flow controlled ventilation in Acute Respiratory Distress Syndrome associated with COVID-19: A structured summary of a study protocol for a randomised controlled trial. Trials, 21(1), 781. https://pubmed.ncbi.nlm.nih.gov/32917259/

Romano, T. G., Mendes, P. V., Park, M., \& Costa, E. L. V. (2017). Extracorporeal respiratory support in adult patients. Jornal Brasileiro de Pneumologia [online], 43(1), 60-70. https://www.scielo.br/j/jbpneu/a/xDMfv6JJQfTchN5Hw3QS7jb/abstract/?lang=pt

Shaefi, S., Brenner, S. K., Gupta, S., O’Gara, B. P., Krajewski, M. L., Charytan, D. M., Chaudhry, S. et al. (2021). Extracorporeal membrane oxygenation in patients with severe respiratory failure from COVID-19. Cuidados Intensivos Med, 47, 208-221. https://link.springer.com/article/10.1007/s00134-020-06331-9

Sousa, M. N. A., De Estrela, Y., De C. A., \& Bezerra, A. L. D. (2020) Perfil epidemiológico de casos de coronavírus no Estado da Paraíba utilizando o Boletim Epidemiológico local. Informação em Pauta, 5(2), 91-106. http://www.periodicos.ufc.br/informacaoempauta/article/view/44288

Verdejo, C., Vergara-Merino, L., Meza, N., Pérez-Bracchiglione, J., Carvajal-Juliá, N., Madrid, E., Rada. G., \& Reyes, M, X, R. (2020). Macrolides for the treatment of COVID-19: a living, systematic review. Medwave, 20(11). https://doi.org/10.5867/medwave.2020.11.8073

Wu, Z., \& McGoogan J. M. (2020). Characteristics of and Important Lessons From the Coronavirus Disease 2019 (COVID-19) Outbreak in China: Summary of a Report of 72314 Cases From the Chinese Center for Disease Control and Prevention. JAMA, 323 (13), 1239-1242. https://pubmed.ncbi.nlm.nih.gov/32091533/ 